\title{
N6-Methyladenosine Induced miR-34a-5p Promotes TNF- $\alpha$-Induced Nucleus Pulposus Cell Senescence by Targeting SIRT1
}

\author{
Hao Zhu',2t, Bao Sun ${ }^{1+}$, Liang Zhu', Guoyou Zou' and Qiang Shen ${ }^{1 *}$ \\ ${ }^{1}$ Department of Orthopaedics, The Affiliated Shanghai General Hospital of Nanjing Medical University, Shanghai, China, \\ ${ }^{2}$ Department of Orthopaedics, Yancheng First Hospital, Affiliated Hospital of Nanjing University Medical School, Yancheng, \\ China
}

\section{OPEN ACCESS}

Edited by:

Roland Wohlgemuth,

Lodz University of Technology, Poland

Reviewed by:

Daniela Carlisi,

University of Palermo, Italy

Wenbin Hua,

Huazhong University of Science

and Technology, China

William K. K. Wu,

Chinese University of Hong Kong,

China

*Correspondence:

Qiang Shen

shenshsy@163.com

${ }^{\dagger}$ These authors have contributed equally to this work

Specialty section:

This article was submitted to

Molecular Medicine,

a section of the journal

Frontiers in Cell and Developmental

Biology

Received: 16 December 2020

Accepted: 15 February 2021

Published: 05 March 2021

Citation:

Zhu H, Sun B, Zhu L, Zou G and

Shen Q (2021) N6-Methyladenosine Induced miR-34a-5p Promotes

TNF- $\alpha$-Induced Nucleus Pulposus

Cell Senescence by Targeting SIRT1.

Front. Cell Dev. Biol. 9:642437.

doi: 10.3389/fcell.2021.642437
Low back pain is tightly associated with intervertebral disc degeneration (IVDD) and aberrant nucleus pulposus (NP) is a critical cause. miRNAs N6-methyladenosine (m6A) modification accounts for the TNF- $\alpha$-induced senescence of NP cells. The aim of this study was to investigate whether m6A modification regulates TNF- $\alpha$-mediated cell viability, cell cycle arrest, and cell senescence and how it works. The results showed that METTL14 expression positively correlated with m6A and TNF- $\alpha$ expression in HNPCs. The knockdown of METTL14 led to the inhibition of the TNF- $\alpha$-induced cell senescence. METTL14 overexpression promoted cell senescence. METTL14 regulated the m6A modification of miR-34a-5p and interacted with DGCR8 to process miR-34a-5p. The miR-34a-5p inhibitor inhibited the cell cycle senescence of HNPCs. miR-34a-5p was predicted to interact with the SIRT1 mRNA. SIRT1 overexpression counteracted the miR-34a-5p-promoted cell senescence. METTL14 participates in the TNF- $\alpha$-induced m6A modification of miR-34a-5p to promote cell senescence in HNPCs and NP cells of IVDD patients. Downregulation of either METTL14 expression or miR-34a-5p leads to the inhibition of cell cycle arrest and senescence. SIRT1 mRNA is an effective binding target of miR-34a-5p, and SIRT1 overexpression mitigates the cell cycle arrest and senescence caused by miR-34a-5p.

Keywords: N6-methyladenosine, IVDD, miR-34a-5p, cell senescence, SIRT1

\section{INTRODUCTION}

Lower back pain (LBP) is the most common chronic pain that affects at least $80 \%$ of Americans in their lifetime (Freburger et al., 2009). In general, LBP can be caused by a muscle sprain or strain injury as well as certain diseases, including spinal cord cancer, ruptured or herniated disc sciatica arthritis, kidney infections, and spine infections. It has been revealed that LBP is strongly associated with intervertebral disc degeneration (IVDD) and degenerative disc disease is identified to be the main cause of LBP (Luoma et al., 2000; Morgan et al., 2014). However, despite some reports of treatment to mitigate the pain and symptoms, no effective therapeutic regimen toward IVDD has been established considering that LBP is multifactorial (Froud et al., 2014). 
Intervertebral disc mainly comprises of inner nucleus pulposus (NP) and surrounded annulus fibrosus, in which NP is the inner core of the vertebral disc (Pattappa et al., 2012). Comprised of a jelly-like material mainly formed by water and a loose collagen fiber network, NP is essential to maintain intervertebral disc height and mechanical properties (Frost et al., 2019). NP is also characterized to be responsible for controlling the synthesis and decomposition of the NP extracellular matrix (ECM) (Hwang et al., 2014). Moreover, abnormal apoptosis of NP cells (NPCs) is correlated with the pathology of IVDD. The degeneration was mainly manifested by the apoptosis of NP cells, in which the levels of cleaved caspase- 3 and Bax were upregulated while the expression of Bcl-2 was downregulated (Kepler et al., 2013; Jiao et al., 2018; Li Z. et al., 2019). Aberrant apoptosis and senescence of NP cells play significant roles in the process of IVDD (Zhao et al., 2006; Jiang et al., 2013; Chen et al., 2018). During the apoptosis of NPCs, tumor necrosis factor (TNF)$\alpha$ represents a key pro-inflammatory cytokine to promote the induction of apoptosis (Xie et al., 2019). Thus, TNF- $\alpha$ has been commonly recognized as a contributor to IVDD (Wang et al., 2017). Additionally, researchers have unveiled the role of TNF$\alpha$ in the premature senescence of rat NP cells (Li P. et al., 2017). Through the PI3K/Akt signaling, TNF- $\alpha$ promoted senescence of NP cells.

Non-coding RNAs (ncRNAs), including microRNAs (miRNAs), long non-coding RNAs (lncRNAs), and circular RNAs, have critical contributions to IVDD (Li et al., 2018; Zhu et al., 2019; Xiang et al., 2020). They participate in the regulation of the proliferation of human NP cells (HNPCs) and the synthesis of ECM as well as the degradation-regeneration balance of the ECM in IVDD. Recently, a comprehensive analysis of altered methylation level of miRNA and InRNAs in IVDD patients was conducted, which verified that N6-methyladenosine (m6A) methylation was one of the most abundant internal RNA modifications in IVDD (Wang X. et al., 2020). A growing number of studies have characterized the critical role of mRNA m6A modification in human diseases (Weng et al., 2018; Han et al., 2019; Han et al., 2020). However, the role of this modification in the pathogenesis of IVDD is fully mysterious. Additionally, the silent mating type information regulator 2 homolog-1 (SIRT1) played a protective role in IVDD and preserves the normal NP cell phenotype (Zhang et al., 2011; Feng et al., 2016; Wang Y. et al., 2020). The mechanism behind has not been explicated yet. In this study, we report the Methyltransferase like (METTL)14dependent m6A methylation of miR-34a-5p in IVDD patients. Through regulating the processing of miR-34a-5p that targeted SIRT1, METTL14-dependent m6A methylation promoted the TNF- $\alpha$-induced cell senescence of human NP cells (HNPCs). Our research provided novel insights into the mechanism of IVDD development.

\section{MATERIALS AND METHODS}

\section{Clinical Sample Collection}

The study was approved by the medical ethics committee of The Affiliated Shanghai General Hospital of Nanjing Medical
University and was conducted in accordance with the Declaration of Helsinki. Written informed consents were obtained from all participants. From March 2015 to April 2018, degenerative nucleus pulposus (NP) samples were collected from 30 patients with intervertebral disc degeneration (IVDD) of low (0-3), moderate (4-6), and high (8-10) degenerative grades $(n=10$ per group) using the Rutgers score (Nakazawa et al., 2018), who underwent operations at The Affiliated Shanghai General Hospital of Nanjing Medical University. Normal NP samples were collected as controls from ten volunteers. The NP tissues were prepared using the culture medium followed by washing with phosphate-buffered saline under sterile conditions. The prepared samples were then used for the qRT-PCR assay.

\section{Cell Culture and Treatment}

HNPCs were isolated and cultured as per the previous report (Dudek et al., 2017). Annulus fibrosus was first washed with sterile phosphate-buffered saline (PBS) 3 times and then meticulously eliminated from the human intervertebral disc tissues. The medium was changed every 3 days. Cultured cells were passaged when reaching a confluence of $80-90 \%$. Cells of the second or third generation were used for further assays. Cells were treated with different doses of TNF- $\alpha$ (SRP2102; SigmaAldrich, St Louis, MO, United States) for $24 \mathrm{~h}$ at $37^{\circ} \mathrm{C}$ and $95 \%$ humidity in an atmosphere of $5 \% \mathrm{CO}_{2}$. Cells without treatment were used as control.

\section{Cell Transfection}

pLKO.1 lentiviral vectors containing shRNA targeting human METTL14 were synthesized by Sangon Biotech (Shanghai, China). Human METTL14 or SIRT1 ectopic expression vector was constructed using the pLVX-Puro or pcDNA3.1(+) vector, respectively. HEK293T cells were cultured in 6-well plates and those were transfected with pLKO.1-METTL14shRNA (shRNA), pLVX-Puro-METTL14 (METTL14), or pcDNA3.1(+)-SIRT1 (SIRT1) using the Lipofectamine reagent as per the manufacturer's protocol. Post-transfection shRNA and METTL14 vectors were collected and were used for transduction. Cells without transduction or transfection were used as control. Cells transduced with pLKO.1-scramble shRNA (shNC), blank pLVX-Puro, or transfected with blank pcDNA3.1(+) vector were used as the negative control.

miR-34a-5p mimic (5'-UGGCAGUGUCUUAGCUGGUUGU$\left.3^{\prime}\right), \quad$ miR-34a-5p inhibitor (5'-ACAACCAGCUAAGACA CUGCCA-3'), and miR-34a-5p negative control (NC, $5^{\prime}$ CAGUACUUUUGUGUAGUACAA- $3^{\prime}$ ) were synthesized by Genepharm Technologies (Shanghai, China). Transfection was performed using Lipofectamine 2,000 (Invitrogen) as per the manufacturer's instructions. Cells without transfection were used as control.

\section{Cell Cycle Assay}

After treatment, HNPCs were spun down at $1,000 \times \mathrm{g}$ for $5 \mathrm{~min}$ and fixed with $700 \mu \mathrm{L}$ of pre-cooled absolute ethanol. RNase A $(1 \mathrm{mg} / \mathrm{mL}, 100 \mu \mathrm{L})$ was added to the fixed cells for 30 min incubation 1 in darkness. The resulting cells were further stained with $50 \mu \mathrm{g} / \mathrm{mL}$ of propidium iodide (PI, $400 \mu \mathrm{L}$ ) for 
10 min. FACScan flow cytometry (Becton Dickinson, Franklin Lakes, NJ) was then performed using the Cell Quest software (Becton Dickinson).

\section{SA $\beta$-Galactosidase Staining}

After treatment, HNPCs were washed with PBS 3 times and stained with freshly prepared SA- $\beta$-Gal staining solution following the manufacturer's protocol (Beyotime Biotechnology Ltd., Shanghai, China). The stained cells were then observed under a microscope.

\section{Co-immunoprecipitation}

Cell lysates were prepared with the Radioimmunoprecipitation assay (RIPA) lysis buffer. Antibodies, including anti-METTL14 (ab252562; Abcam, Cambridge, MA, United States), anti-DGCR8 (ab90579; Abcam) or normal IgG antibody (sc-2027; Santa Cruz Biotechnology, Inc.), were incubated with the cell lysates followed by incubation with Protein A/G PLUS-Agarose beads (sc-2003; Santa Cruz Biotechnology, Inc.) for $2 \mathrm{~h}$ at $4^{\circ} \mathrm{C}$. After washing with the lysis buffer 3 times, the samples were subjected to Western blot analysis using anti-METTL14 (ab223090; Abcam) and antiDGCR8 (ab191875; Abcam) antibodies.

\section{m6A Content Analysis}

$\mathrm{m}^{6} \mathrm{~A}$ in total RNA was analyzed with the m6A RNA Methylation Assay Kit as per the manufacturer's protocol (Abcam, ab185912).

\section{RNA Immunoprecipitation Assays}

Using the Magna RIP RNA-Binding Protein Immunoprecipitation kit (Millipore), RNA immunoprecipitation (RIP) assays were carried out following the manufacturer's protocol. Total RNA (input control) and isotype control (IgG) were detected simultaneously. RNAs were extracted for reverse transcription and qRT-PCR.

\section{Luciferase Reporter Assay}

SIRT1 3'-UTR region containing a putative miR-34a-5p binding site was cloned into the pGL3 vector. For SIRT1 luciferase reporter assay, the HNPCs were transfected with miR-34a-5p mimic, miR-34a-5p inhibitor, and pGL3-SIRT1-WT or pGL3SIRT1-MUT plasmid and pRL-TK vector (Promega) expressing the renilla luciferase with Lipofectamine 2,000. The relative luciferase activity was determined and normalized to the Renilla luciferase activity $48 \mathrm{~h}$ after transfection according to the standard protocol.

\section{RNA Isolation and Quantitative RT-PCR}

Total RNA of HNPCs was extracted using TRIzol reagent (Invitrogen) and the RNeasy Mini Kit (Qiagen, Hilden, Germany). Reverse transcription was conducted using PrimeScript ${ }^{\mathrm{TM}} \mathrm{RT}$ reagent Kit with gDNA Eraser (Takara, Beijing, China). The resulting products were used for qRTPCR amplification with SYBR Premix Ex Taq ${ }^{\mathrm{TM}}$ GC (Takara). GAPDH or U6 was used as the normalization control. The primers for qRT-PCR are listed in Table 1. The fold changes of mRNA or miRNA were determined by the $2^{-\Delta \Delta C T}$ method.
TABLE 1 | Primes sequences used in this study.

\begin{tabular}{|c|c|}
\hline Gene & Sequences $\left(5^{\prime}-3^{\prime}\right)$ \\
\hline TNF- $\alpha$-forward & GGTATGAGCCCATCTATCTG \\
\hline TNF- $\alpha$-reverse & AGGGCAATGATCCCAAAG \\
\hline METTL3-forward & CCTIGCCAGTTCGTTAGTC \\
\hline METTL3-reverse & TCCTCCTTGGTTCCATAGTC \\
\hline METTL14-forward & CTGGGAATGAAGTCAGGATAG \\
\hline METTL14-reverse & CCAGGGTATGGAACGTAATAG \\
\hline WTAP-forward & AAAGCAGTGAGTGGGAAAG \\
\hline WTAP-reverse & AGCGGCAGAAGTATTGAAG \\
\hline SIRT1-forward & АССТССТСАTTGTTATTGG \\
\hline SIRT1-reverse & TTACAGGGTTACAGCAAAG \\
\hline pri-miR-34a-forward & AGTTGCTGAAGGTGGTGGTC \\
\hline pri-miR-34a-reverse & ACATGCGTGCCTGTAGTCC \\
\hline GAPDH-forward & AАTCCCATCACCATCTTC \\
\hline GAPDH-reverse & AGGCTGTTGTCATACTTC \\
\hline miR-200c-3p-forward & CGCGTAATACTGCCGGGTAAT \\
\hline miR-200c-3p-reverse & AGTGCAGGGTCCGAGGTATT \\
\hline miR-27a-3p-forward & GCGCGTTCACAGTGGCTAAG \\
\hline miR-27a-3p-reverse & AGTGCAGGGTCCGAGGTATT \\
\hline miR-34a-5p-forward & CGCGTGGCAGTGTCTTAGCT \\
\hline miR-34a-5p-reverse & AGTGCAGGGTCCGAGGTATT \\
\hline miR-15b-5p-forward & CGCGTAGCAGCACATCATGG \\
\hline miR-15b-5p-reverse & AGTGCAGGGTCCGAGGTATT \\
\hline pre-miR-34a-forward & CCTAGAAGTGCTGCACGTTGTG \\
\hline pre-miR-34a-forward & AGTGCAGGGTCCGAGGTATT \\
\hline U6-forward & CTCGCTTCGGCAGCACA \\
\hline U6-reverse & AACGCTTCACGAATTTGCGT \\
\hline
\end{tabular}

\section{Western Blot}

The cell lysates were prepared using the radioimmunoprecipitation assay lysis buffer (Beyotime, Shanghai, China). The quantitation of protein in the samples was performed using the bicinchoninic acid (BCA) Protein Assay Kit (Beyotime). For SDS-PAGE, $20 \mu \mathrm{g}$ of protein sample was loaded to each well followed by the transmembrane onto polyvinylidene fluoride membranes (Millipore, Billerica, MA, United States). The blocking of the membrane was conducted by incubating with 5\% fat-free milk and then primary antibodies against METTL3 (ab195352; Abcam), METTL14 (ab223090; Abcam), WTAP (ab195380; Abcam), SIRT1 (ab110304; Abcam), and GAPDH (\#5174, Cell Signaling Technology) overnight at $4^{\circ} \mathrm{C}$. The blots were then washed in Tris-buffered Saline Tween-20 (TBST) three times and then incubated with secondary antibodies (A0208, A0216; Beyotime, Shanghai, China). The blots were examined by chemiluminescence using the Enhanced Chemiluminescence Detection kit (Pierce Biotechnology, Rockford, IL, United States). After exposure, the intensity of bands was analyzed by Image-Pro Plus 6.0 software.

\section{Statistical Analysis}

All statistical analyses were performed using GraphPad Prism 8.0.2 (GraphPad Software, San Diego, CA, United States). Data were shown as mean \pm standard deviation (SD) from the triplicates of independent experiments. The difference between 
different groups was analyzed using a two-sided Student's $t$-test and ANOVA. $P$-values $<0.05$ were considered significant.

\section{RESULTS}

\section{The Level of m6A Modification Is Positively Correlated With TNF- $\alpha$ in IVDD Patients}

TNF- $\alpha$ is the main pro-inflammatory cytokines principally associated with the progression of IVDD (Purmessur et al., 2013). To verify the relationship between TNF- $\alpha$ and the m6A methylation in IVDD patients, we first examined the level of m6A methylation in the NP tissues from the patients and discovered an increase in the level of $\mathrm{m} 6 \mathrm{~A}$ modification (Figure 1A). Therefore, we further implemented screening of the expression of genes related to $\mathrm{m} 6 \mathrm{~A}$ methylation, including METTL3, METTL14, and Wilms tumor 1-associated protein (Figures 1B-D). With the increase of the IVDD grades, all the genes exhibited higher expression than the normal group. Meanwhile, TNF- $\alpha$ expression increased along with the IVDD grades (Figure 1E). To better determine the correlation between the expression of TNF- $\alpha$ and m6A modification-related genes, we calculated Pearson correlation coefficients for each gene by plotting the TNF- $\alpha$ mRNA level against the m6A level or the mRNA level of the genes (Figures 1F-I). Among the three genes, TNF- $\alpha$ and METTL14 $(r=0.7299, P<0.001)$ were more correlated than the other two, which is similar to the correlation between TNF- $\alpha$ expression and the m6A methylation level. Similar phenomena were also observed in HNPCs. With the increase in the stimulation concentrations of TNF- $\alpha$, the level of m6A methylation was significantly elevated (Figure 1J). Accordingly, compared with the untreated HNPCs, the METTL14 mRNA level and the protein level in the treatment groups had also experienced significant increases (Figures $1 \mathrm{~K}, \mathbf{L}$ ). As a result, it is indicated that the METTL14mediated m6A modification is positively correlated with TNF$\alpha$ in IVDD models.

\section{METTL14 Knockdown Inhibits TNF- $\alpha$-Induced Cell Cycle Arrest and Senescence}

The discovery of the positive correlation between the expression of METTL14 and TNF- $\alpha$ in IVDD models motivated us to further explore the impact of METTL14 on TNF- $\alpha$-induced NP cellular processes. We developed three shRNAs (shRNA 1-3) to silence METTL14 in HNPCs, which significantly reduced the expression of the mRNA and protein compared with the controls (Figures 2A,B). Especially, shRNA 1 and 2 showed better inhibitory potency according to the Western blot result (Figure 2B). In the cell viability assay, TNF- $\alpha$ treatment reduced the cell viability while the use of the two shRNAs to TNF- $\alpha$-treated HNPCs significantly restored the cell viability (Figure 2C). A further investigation indicated that the suppression of the METTL14 expression affected the TNF- $\alpha$ induced cell cycle arrest (Figures 2D,E). TNF- $\alpha$ caused the cell cycle arrest of HNPCs at the G0-G1 stage. In comparison, shRNA1 and 2 significantly drove the cells to enter the $S$ and G2$\mathrm{M}$ phases. Additionally, the knockdown of METTL14 enabled the inhibition of TNF- $\alpha$-induced cell senescence (Figure 2F). Using a senescence-associated (SA) $\beta$-galactose assay, we observed that the blue-dyed precipitates had been considerably diminished in the shRNA-treatment groups. From the results above, it is suggested that METTL14 knockdown significantly inhibits TNF- $\alpha$-induced cell viability inhibition, cell cycle arrest, and cell senescence.

\section{METTL14 Overexpression Promotes Cell Cycle Arrest and Senescence}

To further evaluate the influence of METTL14 on cell cycle arrest and senescence, we overexpressed METTL14 in HNPCs without the TNF- $\alpha$ treatment (Figures 3A,B). Interestingly, despite the absence of TNF- $\alpha$, the overexpression of METTL14 led to reduced cell viability (Figure 3C). Likewise, METTL14 overexpression remarkably influenced the cell cycle (Figures 3D,E). METTL14 overexpression enhanced the cell cycle arrest, which brought up the percentage of the cells of the G0-G1 phase by 20\% (50\% for Vector vs. $70 \%$ for METTL14). Accordingly, the cell percentage of $S$ and G2-M phases declined. With regard to the efficiency, the overexpression of METTL14 was even comparable to the TNF- $\alpha$ treatment (Figures 3D,E). When using the SA $\beta$ galactose assay to identify the cell senescence of METTL14overexpressing HNPCs, we observed promoted cell senescence (Figure 3F), which was similar to the TNF- $\alpha$ treatment. Therefore, the METTL14 overexpression assays substantiated that METTL14 inhibited cell viability and promote cell cycle arrest and senescence.

\section{METTL14-Dependent m6A Methylation Regulates the Processing of miR-34a by DGCR8}

METTL14 can interact with DGCR8 to positively regulate the primary miRNA process in an m6A-dependent manner (Ma et al., 2017). In our case, we intended to pursue the downstream target of the METTL14-DGCR8 axis. To this end, we first established the interaction between DGCR8 and METTL14 in HNPCs by co-immunoprecipitation (Figure 4A). Moreover, in METTL14overexpressing HNPCs we also saw a significant increase in the binding between METTL14 and DGCR8 (Figure 4B). The findings above solidified that METTL14 mediated primiRNA processing by regulating the recognition and binding of DGCR8 to pri-miRNAs.

Furthermore, we detected the association between the four miRNAs, which were upregulated in TNF- $\alpha$-treated HNPCs and in NP tissues from IVDD patients (Cao and Chen, 2017; Kang et al., 2017; Cheng et al., 2018; Xiang et al., 2020), and METTL14 (Figure 4C). Compared with the other three miRNAs, the miR-34a-5p level skyrocketed (>3.5-fold) accompanying the overexpression of METTL14 (Figure 4D). Accordingly, the suppression of METTL14 

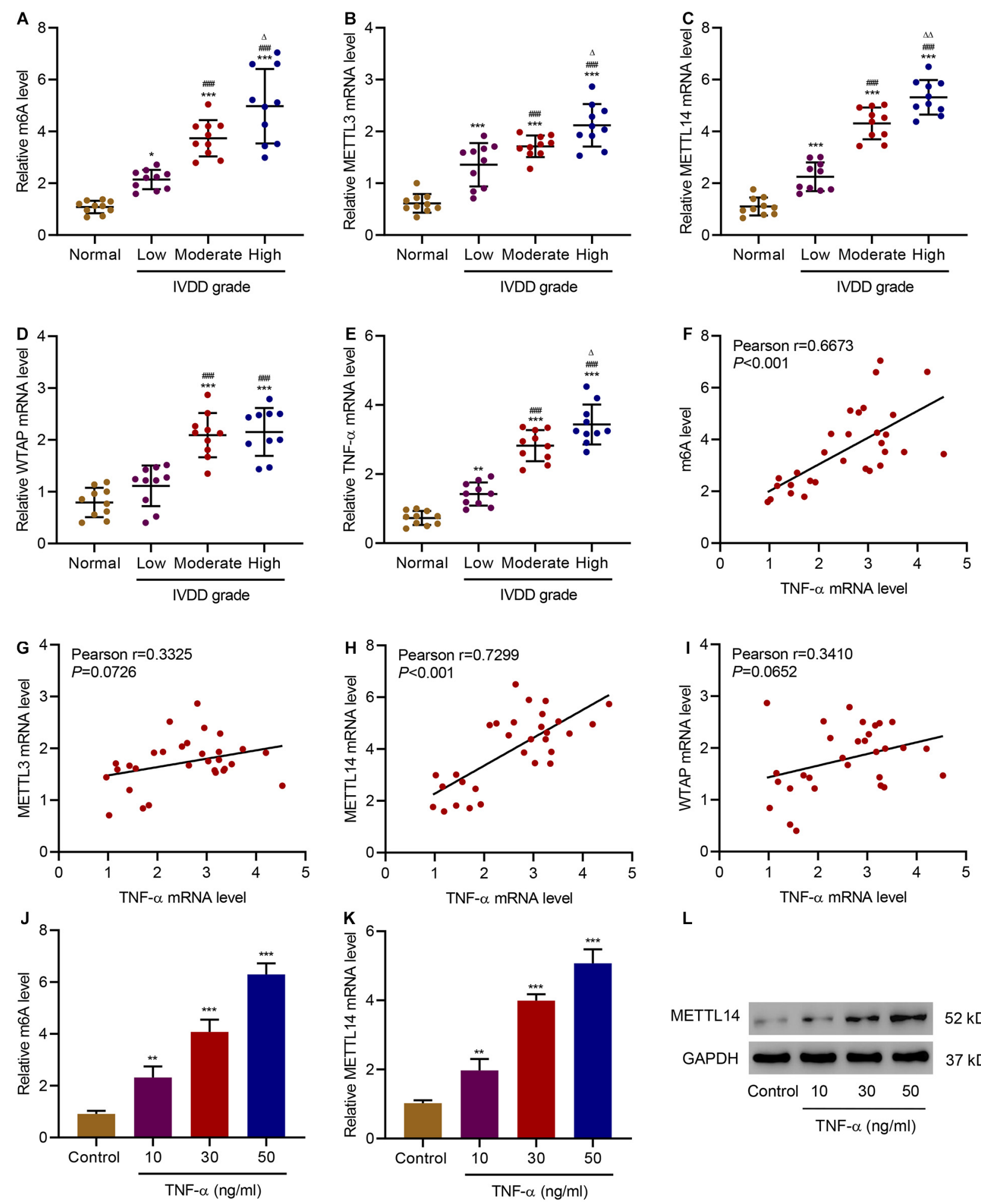

$\mathbf{L}$

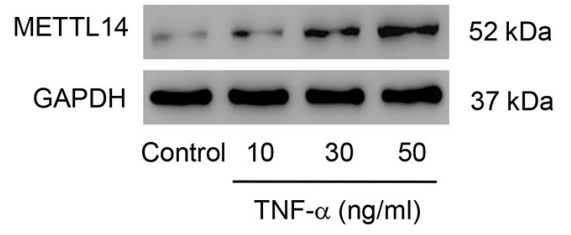

FIGURE 1 | Correlation between TNF- $\alpha$ and m6A modification in IVDD patients. (A) The m6A level and expression of (B) METTL3, (C) METTL14, (D) WTAP, and (E) TNF- $\alpha$ in normal controls and IVDD patients. (F-I) Pearson correlation scatter plots in IVDD patients $(n=30)$. (J) The m6A level and $(\mathbf{K}, \mathbf{L})$ expression of METTL14 in HNPCs treated with different doses of TNF- $\alpha .{ }^{\star} P<0.05,{ }^{\star \star} P<0.01,{ }^{\star \star \star} P<0.001$ compared with normal control; \#\#\# $P<0.001$ compared with low grade; $\Delta P<0.05, \Delta \Delta P<0.01$ compared with moderate grade. 

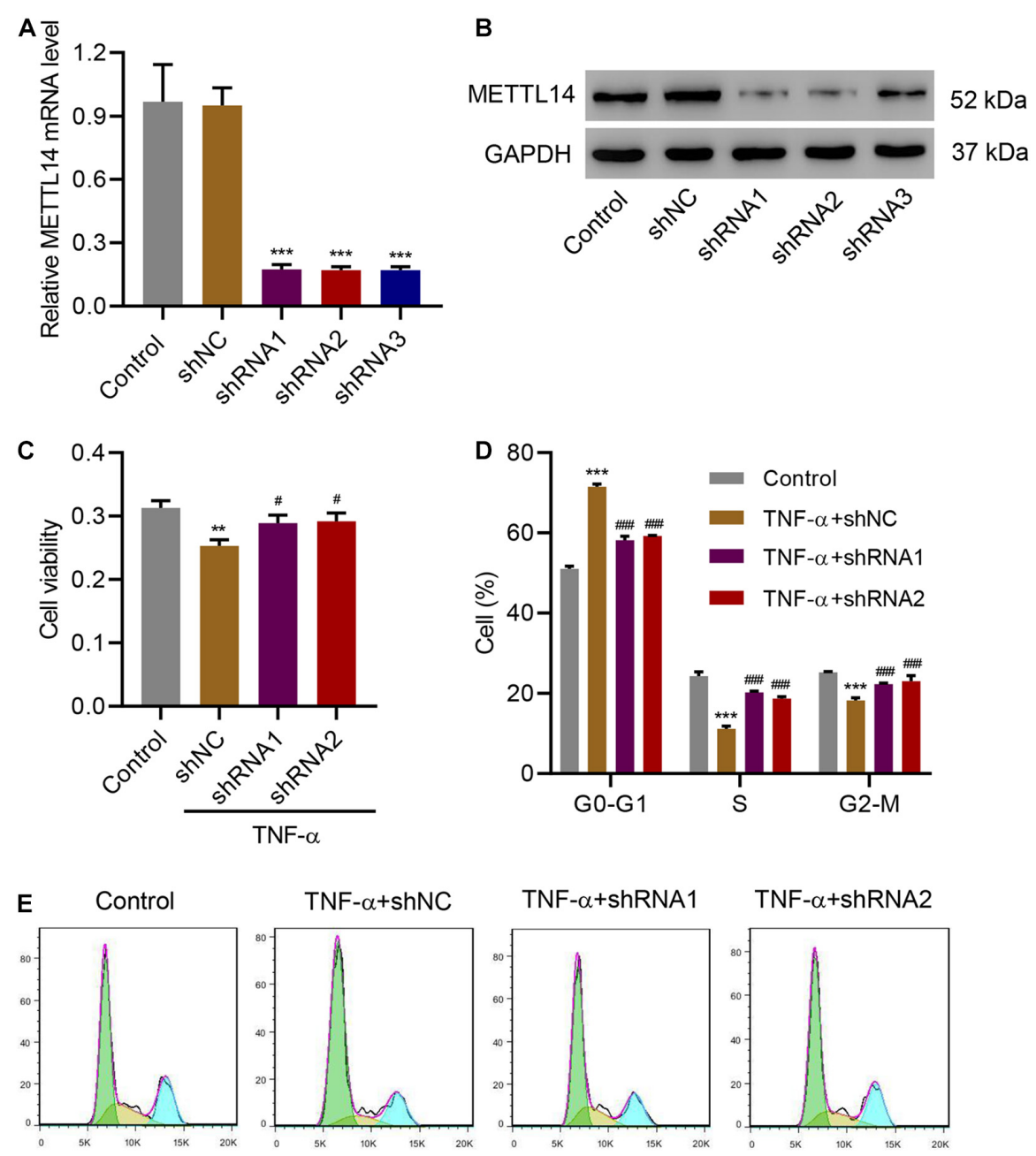

TNF- $\alpha+\operatorname{shRNA2}$
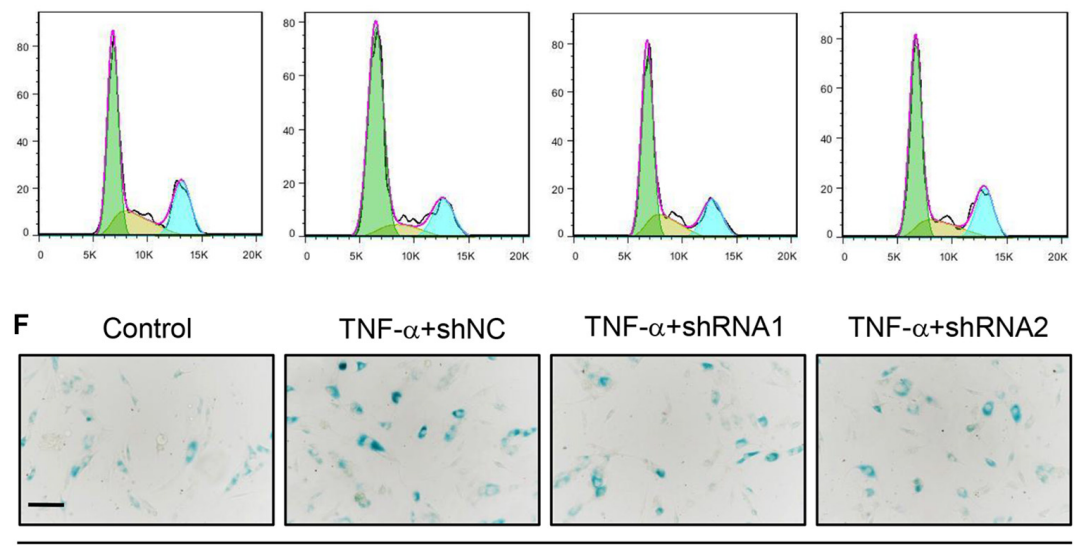

SA- $\beta$-gal

FIGURE 2 | METTL14 silencing inhibits TNF- $\alpha$-induced cell cycle arrest and senescence. (A,B) Expression of METTL14 in HNPCs transduced with METTL14 shRNA vectors. (C) Cell viability, (D,E) cell cycle, and (F) SA- $\beta$-gal staining of HNPCs transduced with METTL14 shRNA vectors and treated with $30 \mathrm{ng} / \mathrm{mL}$ TNF- $\alpha$ for $24 \mathrm{~h}$.

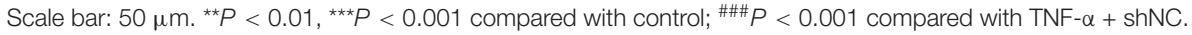

by the specific shRNAs significantly reduced the miR-34a$5 \mathrm{p}$ relative level. When subjected to the TNF- $\alpha$ treatment, HNPCs exhibited remarkably high expression of miR-34a$5 \mathrm{p}$ and pre-miR-34 but low level of pri-miR-34, indicating that miR-34 was processed (Figure 4E). The addition of METTL14 shRNAs tremendously mitigated the TNF- $\alpha$-induced increase in mRNA levels of miR-34a-5p and pre-miR-34. Furthermore, the relative pri-miR-34a m6A level was also enhanced by TNF- $\alpha$, which was abolished by METTL14 knockdown (Figure 4F). Conversely, the overexpression of METTL14 promoted the formation of miR-34a-5p and pre-miR-34 (Figure 4G). The relative level of pri-miR-34a m6A showed a fourfold increase when METTL14 was overexpressed (Figure $4 \mathrm{H}$ ).

As a result, the level of METTL14 exhibited a positive correlation with that of miR-34a-5p in NP tissues of IVDD patients (Figure 4I). The regulation of the pri-miR-34a level was tightly associated with the interaction between METTL14 and DGCR8 (Figures 4J,K). The DGCR binding level as well as the m6A modification level was significantly promoted by METTL14, 


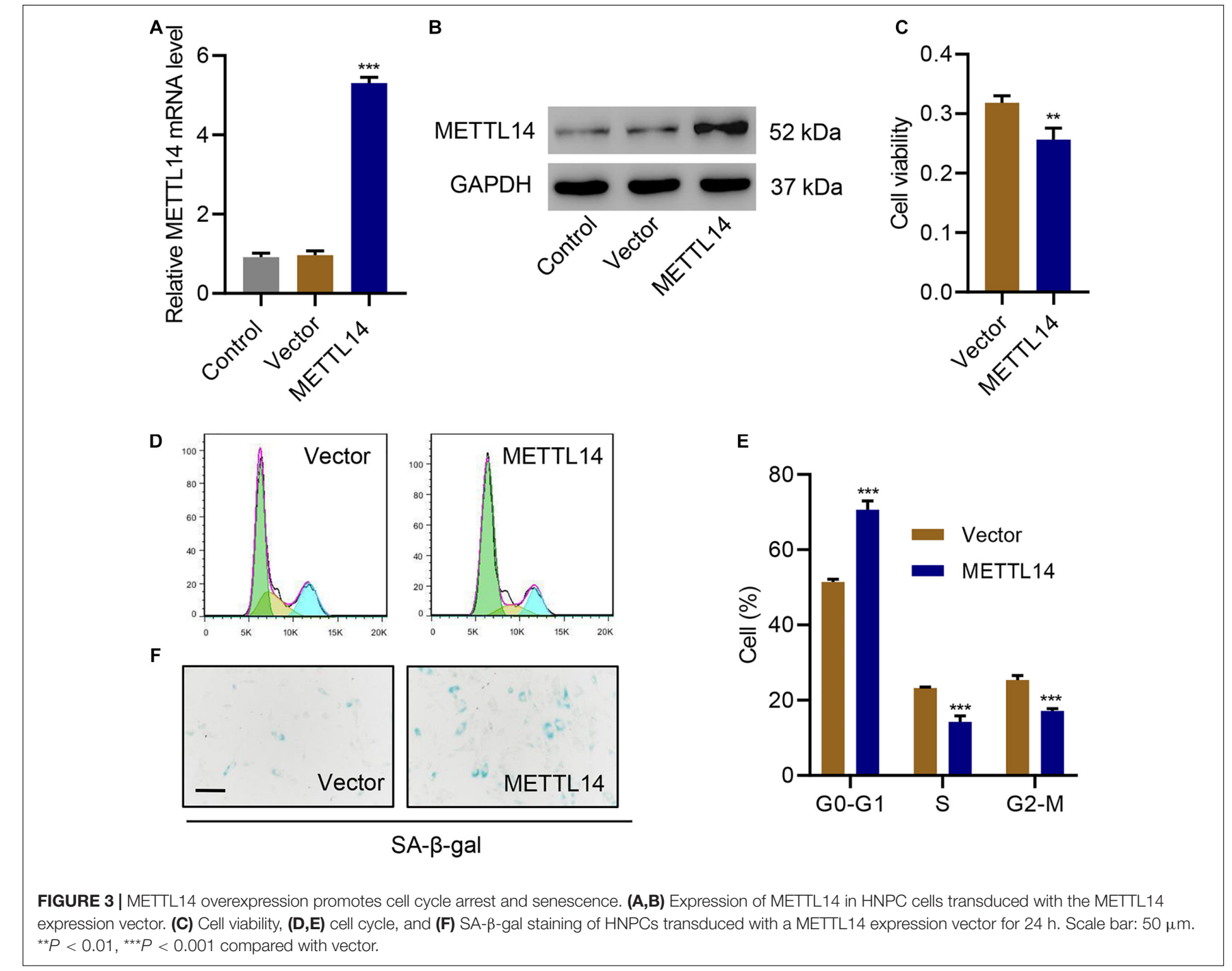

B C

indicating that METTL14 played a vital role in the maturation of pri-miR-34a. Taken together, these results indicate that the METTL14 promoted the processing of pri-miR-34a by DGCR8 in an m6A manner.

\section{miR-34a-5p Inhibitor Rescues the Cell Cycle Arrest and Senescence Induced by METTL14 Overexpression}

To verify the potential function of miR-34a-5p in IVDD, we introduced the corresponding miRNA inhibitor in the METTL14-overexpressing HNPCs. The miR-34a-5p inhibitor considerably counteracted the effects of METTL14 overexpression on cell senescence of HNPCs (Figure 5). The cell viability (Figure 5A), cell cycle arrest (Figures 5B,C), and cell senescence (Figure 5D) were largely rescued by the inhibitor. As a result, evidenced by the data, the METTL14-induced cell cycle arrest and senescence can be recovered by the miR-34a-5p inhibitor, thus manifesting the indispensable role of miR-34a-5p in regulating METTL14-dependent cell senescence.

\section{miR-34a-5p Promotes Cell Cycle Arrest and Senescence by Targeting SIRT1}

Using the mRNA interaction prediction server ${ }^{1}$, we predicted the potential interaction between the miR-34-5p and the $3^{\prime}$-UTR of SIRT1 mRNA (Figure 6A). To better analyze the interaction, we devised a dual-luciferase assay using the SIRT1 wildtype mRNA (SIRT1-WT) and the SIRT1 3'-UTR mutant mRNA (SIRT1-MUT) in HNPCs that were treated with the miR-34a$5 \mathrm{p}$ inhibitor or the miR-34a-5p mRNA mimic (Figure 6B). In the SIRT1-WT group, the inhibitor approximately generated a 9-fold drastic increase in luciferase activity. By contrast, the mimic led to lower luciferase activity. Instead of inducing changes in luciferase activities, in the SIRT1-MUT group, no matter whether the inhibitor or the mimic failed to generate any signal, indicating that the mutation of $3^{\prime}$-UTR jeopardized the interaction between miR34a-5p and SIRT1 mRNA. Furthermore, SIRT1 mRNA expression can be influenced by miR-34a-5p

\footnotetext{
${ }^{1}$ http://www.microrna.org/
} 


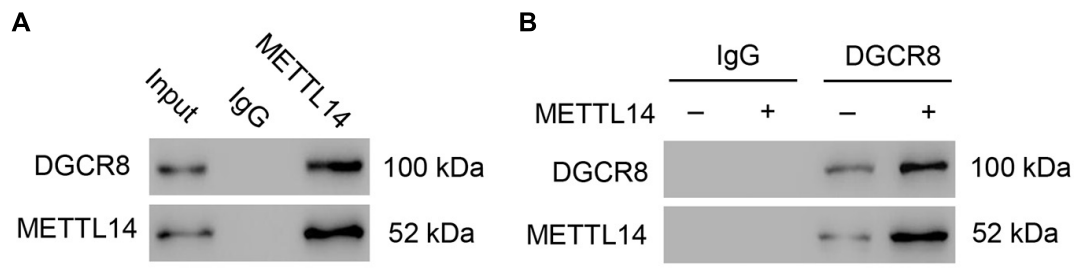

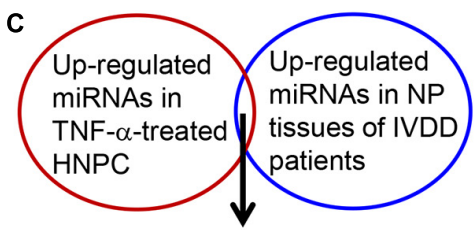

miR-200c-3p, miR-27a-3p, miR-34a-5p, miR-15b
D

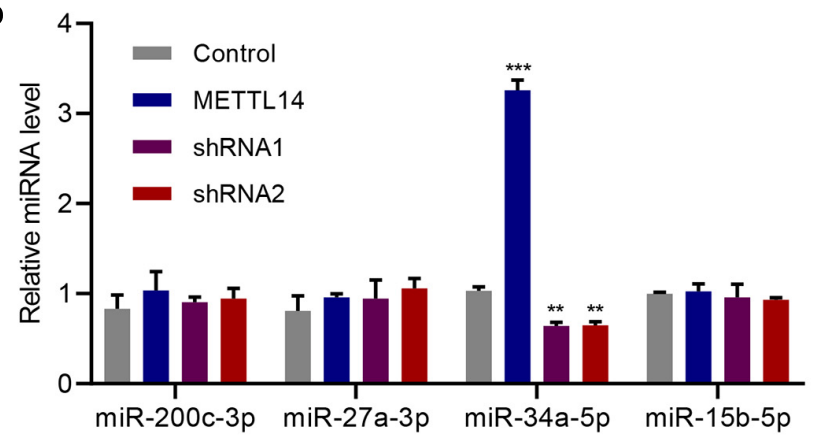

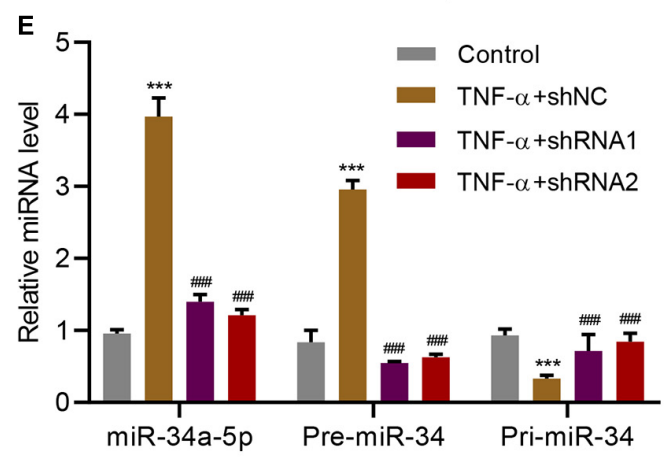

$\mathbf{F}$
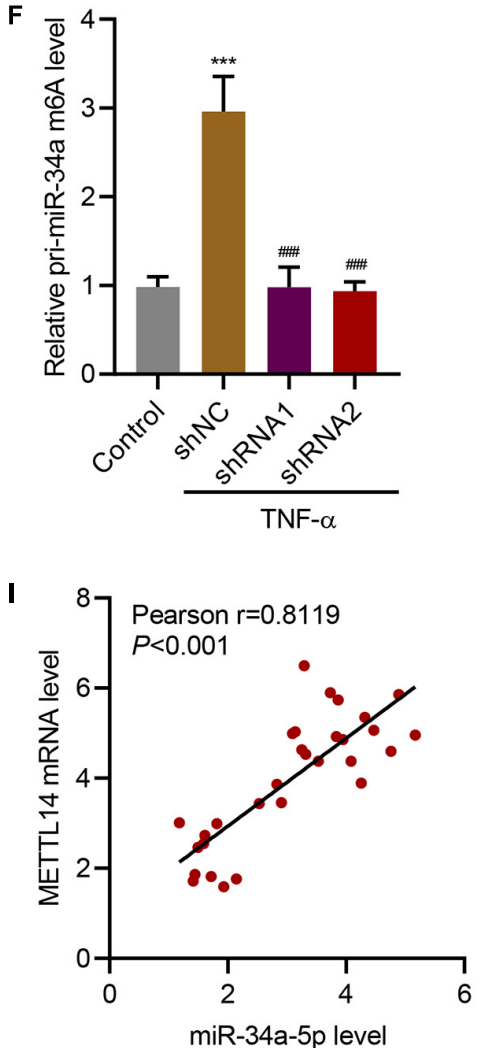
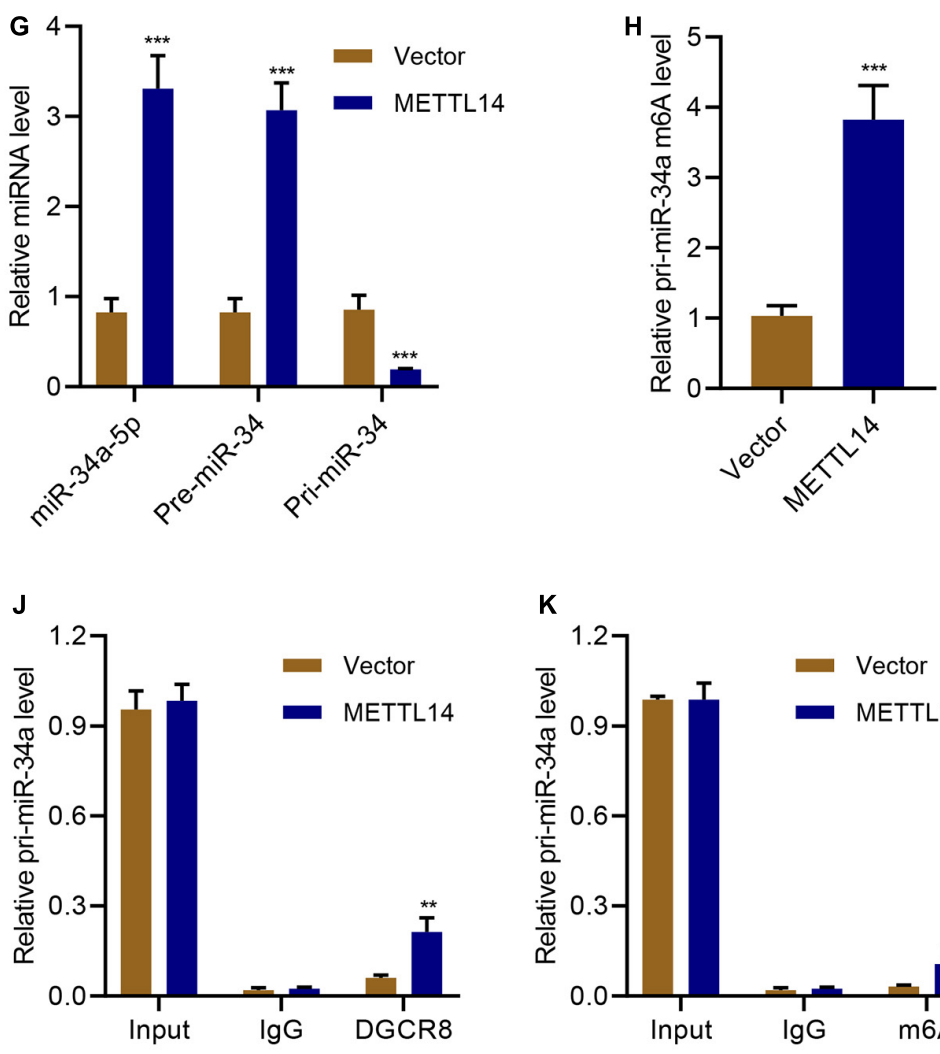

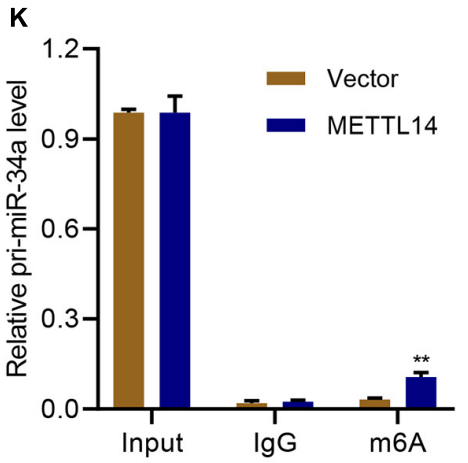

FIGURE 4 | METTL14-dependent m6A methylation regulates the processing of miR-34a by DGCR8. (A) Co-immunoprecipitation of the METTL14-interacting protein DGCR8. IgG antibody was used as the control for the immunoprecipitation. (B) Immunoprecipitation of DGCR8 in cells overexpressing METTL14 or not. Western blot was conducted using the antibodies depicted. (C) Four up-regulated miRNAs were searched out according to their expression levels in TNF- $\alpha$-treated HNPC cells and in NP tissues of IVDD patients. (D) Expression of miR-200c-3p, miR-27a-3p, miR-34a-5p and miR-15b-5p in HNPC cells transduced with METTL14 shRNA vectors or overexpression vector. (E) Expression of pri-miR-34a, pre-miR-34a, and miR-34a-5p and (F) the levels of pri-miR-34a m6A in HNPC cells transduced with METTL14 shRNA vectors and treated with $30 \mathrm{ng} / \mathrm{mL}$ TNF- $\alpha$ for 24 h. (G) Expression of pri-miR-34a, pre-miR-34a, and miR-34a-5p and (H) the levels of pri-miR-34a m6A in HNPC cells transduced with a METTL14 expression vector for $24 \mathrm{~h}$. (I) Pearson correlation scatter plots in IVDD patients $(n=30)$. Immunoprecipitation of (J) DGCR8-associated and (K) m6A modified RNA from HNPC cells transduced with METTL14 expression vector followed by qRT-PCR to detect pri-miR-34 binding to DGCR8 and to assess the pri-miR-34a m6A modification level, respectively. ${ }^{* \star} P<0.01,{ }^{* \star *} P<0.001$ compared with control or vector. $\# \# P<0.001$ compared with TNF- $\alpha+$ shNC. 

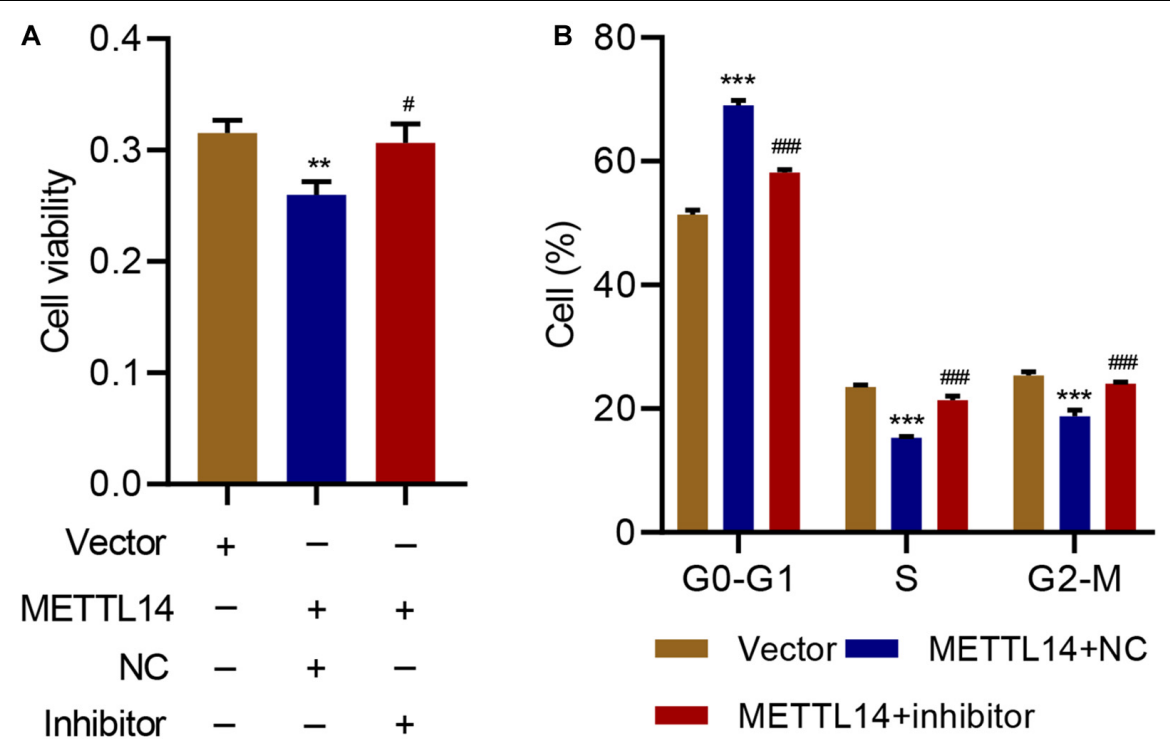

c Vector

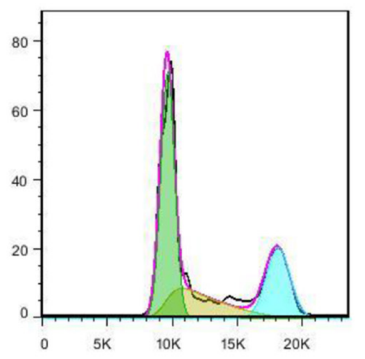

D

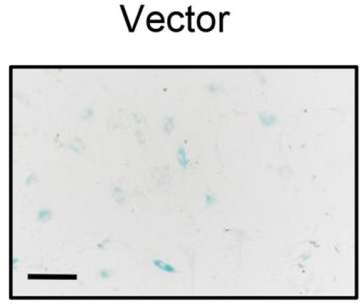

METTL14+NC

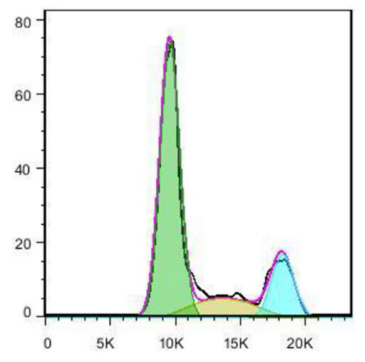

METTL14+NC

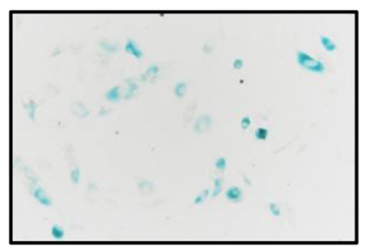

METTL14+inhibitor

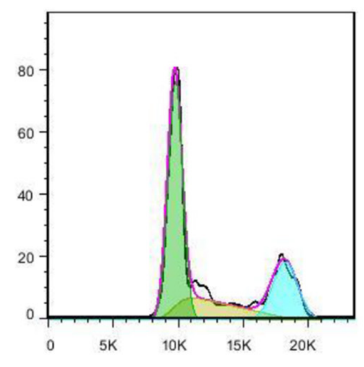

METTL14+inhibitor

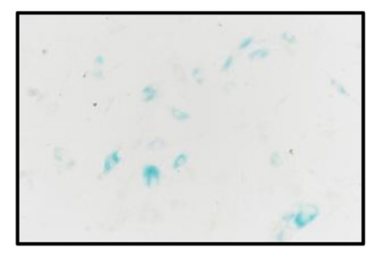

SA- $\beta$-gal

FIGURE 5 | miR-34a-5p inhibitor rescues the cell cycle arrest and senescence induced by METTL14 overexpression. (A) Cell viability, (B,C) cell cycle, and (D) SA- $\beta$-gal staining of HNPC cells transduced with the METTL14 expression vector and transfected with miR-34a-5p inhibitor for $24 \mathrm{~h}$. Scale bar: $50 \mu \mathrm{m}$. ${ }^{* \star} P<0.01,{ }^{* \star} P<0.001$ compared with vector. ${ }^{\# \# \#} P<0.001$ compared with METTL14 + NC.

(Figure 6C). The miR-34a-5p inhibitor significantly promoted SIRT1 expression in HNPCs while the mimic considerably suppressed the expression. We next overexpressed SIRT1 in HNPCs to determine the effects of SIRT1 on cell senescence of HNPCs in the presence of the inhibitor or the mimic of miR-34a-5p (Figures 6D,E). The miR-34a-5p mimic lowered the cell viability of HNPCs. However, SIRT1 overexpression in HNPCs largely restored the cell viability despite the presence of the miR-34a-5p mimic (Figure 6F). SIRT1 overexpression was also active in attenuating the miR-34a-5p-induced cell cycle arrest, in which more HNPCs entered S and G2-M phases (Figure 6G,H). Cell senescence of HNPCs caused by miR-34a$5 \mathrm{p}$ was reversed by SIRT1 overexpression as well (Figure 6I). Based on these findings, we established the relationship between the level of SIRT1 mRNA and the levels of miR34a-5p and METTL14 mRNA in NP tissues of IVDD patients (Figures 6J,K). SIRT1 expression was negatively correlated with either the miR-34a-5p level or the METTL14 mRNA level. 


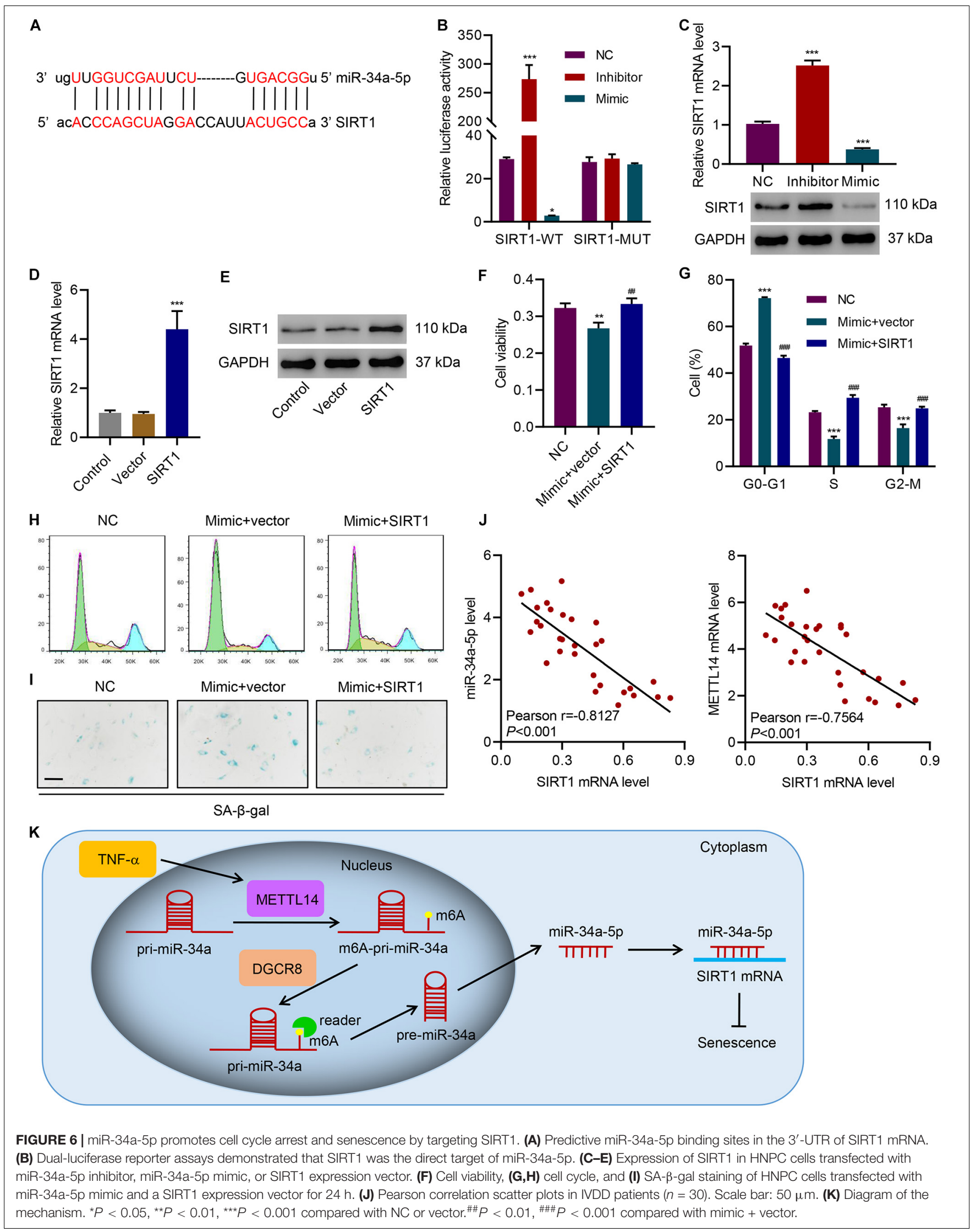


Therefore, our results demonstrate that SIRT1 served as a critical target in process of the miR-34a-5p-promoted cell cycle arrest and senescence.

\section{DISCUSSION}

miRNA has been found closely associated with cellular process regulation, cell function, and diseases (Bhaskaran and Mohan, 2014). The modification of miRNAs, especially methylation, can largely affect the functions of mRNA, which further regulates cellular processes and biological activities (Bianchi et al., 2017). Recent reports link IVDD with miRNAs, suggesting that miRNAs can act as potential therapeutic targets ( $\mathrm{Li}$ et al., 2015; Zhou et al., 2017). Herein, we have devised various assays to clarify the mechanism of m6A methylation promoted cell senescence in IVDD.

The level of m6A modification significantly increased in the HNPCs, which was correlated with the level of TNF- $\alpha$ in IVDD patients. m6A modification has been characterized as the most prevalent internal mRNA modification in mammalian cells, which accounts for regulating various important biological processes (Zhang et al., 2019). Rising evidence is confirming the role of $\mathrm{m} 6 \mathrm{~A}$ in cell development and cancers (Fazi and Fatica, 2019; Chen and Wong, 2020). Li et al. have characterized the function of m6A methylation in controlling the proliferation of human glioma cells by influencing apoptosis (Li F. et al., 2019). Yang et al. have reported the m6A-modulated proliferation and apoptosis of lens epithelial cells (Yang et al., 2020). However, there is no previous report on the relation between m6A methylation and IVDD. Our discovery of a high level of m6A modification in NP tissues of IVDD patients expands the scope of related research. More importantly, we have identified that METTL14, one of the "writer" protein, was more tightly associated with m6A modification, the expression of which was positively correlated with the level of m6A methylation as well as the TNF- $\alpha$. Jian et al. (2020) have reported the mechanism of METTL14-promoted endothelial inflammation and atherosclerosis through driving FOXO1 m6A modifications. They proved the major role of METTL14 in TNF- $\alpha$-induced endothelial cell inflammation. As a process tightly associated with inflammation, cell senescence includes irreversible cell cycle arrest (Stojanovic et al., 2020). In our study, we have clearly illustrated the role of METTL14 in cell senescence, which solidifies the function of METTL14 in TNF- $\alpha$-induced inflammation. With the increased expression of this writer protein, cell viability decreased while cell cycle arrest and senescence were significantly promoted.

The identification of METTL14 as the main regulator of m6A modification in IVDD models allowed us to further explore the mechanism behind it. Studies have shown that METTL14 actively participates in the processing of miRNAs by interacting with DGCR8 (Feng et al., 2010; Ma et al., 2017). Accordingly, our co-immunoprecipitation assay also confirmed the interaction between METTL14 and DGCR8 in HNPCs, which proves that METTL14 played a role in regulating miRNA maturation in IVDD models. Interestingly, through an in-depth screening of miRNA change in either HNPCs or NP tissues of IVDD patients, we identified several upregulated miRNAs, in which miR-34a-5p showed a positive correlation with the m6A modification level. When varying the METLL14 level in HNPCs, we were able to capture the regulation of the miR-34a$5 \mathrm{p}$ processing. Similar to the processing of miR-126 (Feng et al., 2010) and miR-19a (Zhang et al., 2020), METLL14 positively modulated the maturation of miR-34. Thus, the RNA levels of pre-miR-34 and miR-34a-5p were significantly elevated in contrast to the mitigated pri-miR-34 level. The m6A methylation of pri-miR-34 was found active under the circumstance of METTL14 overexpression.

Previous studies have discussed the mechanism of m6Apromoted cell senescence ( $\mathrm{Li}$ Q. et al., 2017; Wu et al., 2020). The METTL3/METTL14-mediated m6A methylation can enhance p21 expression which is further promoted. oxidative stress-induced cellular senescence (Li Q. et al., 2017). Through interacting with Lamin A, METTL3/14 can be properly localized in the nuclear speckles to achieve the regulatory function ( $\mathrm{Wu}$ et al., 2020). Our study has deepened the current understanding of m6A modification in cell senescence. Mainly regulated by METTL14, m6A-involved cell senescence was originated from the methylation of miR-34a-5p followed by the interaction with the $3^{\prime}$-UTR of the SIRT mRNA. During the process, the maturation of the mRNA was largely promoted, reflected by the escalated levels of miR-34a-5p and pre-miR-34and reduced level of pri-miR-34. The role of METTL3 in the regulation of senescence.

It is noted that miRNAs can play vital roles in cell senescence (Li et al., 2009; Faraonio et al., 2012; Baker et al., 2019). miR34a-5p has been identified as a possible cause of cell senescence in HNPCs in our study. Xia et al. have characterized the mechanism of miR-34a-5p-induced cardiac senescence-related injury. It is shown that miR-34a-5p serves as an exosomal transfer RNA and the inhibition of miR-34a-5p mitigated the prosenescent effect in cardiomyocytes and subsequently alleviated the irreversible cell cycle arrest (Xia et al., 2020). miR-34a$5 \mathrm{p}$ is also found involved in regulating the switch between senescence and apoptosis in non-small cell lung cancer (Gupta et al., 2020). Herein, we have presented that the inhibition of miR-34a-5p significantly decreases the senescence of HNPCs. As a result, miR-34a-5p demonstrates prevalent senescent effects in varieties of tissues and organs, which can be a major biological function of this miRNA.

Additionally, Maes et al. (2009) used a miRNA microarray assay to reveal the upregulation of miR-34 in senescent cells. We advanced the results and proved that this miRNA was further processed through the METTL14-DGCR8 axis. Our data have demonstrated that miR-34a-5p induced cell senescence by targeting the downstream factor SIRT1. SIRT1 localizes in both the nucleus and cytoplasm to function in many crucial biological activities, including lifespan extension, ADP-ribosyltransferase, DNA repair, cell cycle arrest, and cellular senescence (Lee et al., 2019). The sequence of miR-34a-5p was predicted to pair with that of the $3^{\prime}$-UTR of SIRT1. The use of the miR-34a$5 \mathrm{p}$ mimic resulted in typical senescence that was largely inhibited by overexpressing SIRT1. In IVDD patients, SIRT1 expression 
was negatively correlated with the levels of the METTL14 mRNA and miR-34a-5p. Therefore, our findings reinforce the idea that SIRT1 prevents cell senescence.

Our data were mainly obtained from the in vitro model of HNPCs, which can be further explored in in vivo IVDD models. Due to limited studies in the related field, the concrete mechanism of METTL14-mediated m6A modification and maturation of miR-34a has not been fully elucidated. Efficient disruption of the interaction between miR-34a-5p and SIRT1 in both in vitro and in vivo levels to alleviate IVDD still requires more sophisticated and intensive investigations. Additionally, the interaction between METTL14 and DGRC8 is also worth dedicated studies to uncover the entire axis.

Our current study reveals that m6A-modified miR-34a$5 \mathrm{p}$ promotes induced NPC senescence by targeting SIRT1, which represents the first attempt to discover the association between miRNA modification and cell senescence in IVDD models (Figure 6K). By characterizing the role of the miR$34 a-5 p-S I R T 1$ axis in cell senescence, we have proposed a potential direction for developing an IVDD therapy by disrupting the interaction between miR-34a-5p and SIRT1 or inhibiting METTL14.

\section{REFERENCES}

Baker, J. R., Vuppusetty, C., Colley, T., Hassibi, S., Fenwick, P. S., Donnelly, L. E., et al. (2019). MicroRNA-570 is a novel regulator of cellular senescence and inflammaging. FASEB J. 33, 1605-1616. doi: 10.1096/fj.201800965R

Bhaskaran, M., and Mohan, M. (2014). MicroRNAs: history, biogenesis, and their evolving role in animal development and disease. Vet. Pathol. 51, 759-774. doi: $10.1177 / 0300985813502820$

Bianchi, M., Renzini, A., Adamo, S., and Moresi, V. (2017). Coordinated actions of micrornas with other epigenetic factors regulate skeletal muscle development and adaptation. Int. J. Mol. Sci. 18:840. doi: 10.3390/ijms18040840

Cao, Z., and Chen, L. (2017). Inhibition of miR-27a suppresses the inflammatory response via the p38/MAPK pathway in intervertebral disc cells. Exp. Ther. Med. 14, 4572-4578. doi: 10.3892/etm.2017.5053

Chen, M., and Wong, C. M. (2020). The emerging roles of N6-methyladenosine (m6A) deregulation in liver carcinogenesis. Mol. Cancer 19:44. doi: 10.1186/ s12943-020-01172-y

Chen, Y., Zheng, Z., Wang, J., Tang, C., Khor, S., Chen, J., et al. (2018). Berberine suppresses apoptosis and extracellular matrix (ECM) degradation in nucleus pulposus cells and ameliorates disc degeneration in a rodent model. Int. J. Biol. Sci. 14, 682-692. doi: 10.7150/ijbs.24081

Cheng, X., Zhang, G., Zhang, L., Hu, Y., Zhang, K., Sun, X., et al. (2018). Mesenchymal stem cells deliver exogenous miR-21 via exosomes to inhibit nucleus pulposus cell apoptosis and reduce intervertebral disc degeneration. J. Cell Mol. Med. 22, 261-276. doi: 10.1111/jcmm.13316

Dudek, M., Yang, N., Ruckshanthi, J. P., Williams, J., Borysiewicz, E., Wang, P., et al. (2017). The intervertebral disc contains intrinsic circadian clocks that are regulated by age and cytokines and linked to degeneration. Annal. Rheum. Dis. 76, 576-584. doi: 10.1136/annrheumdis-2016-209428

Faraonio, R., Salerno, P., Passaro, F., Sedia, C., Iaccio, A., Bellelli, R., et al. (2012). A set of miRNAs participates in the cellular senescence program in human diploid fibroblasts. Cell Death Differ. 19, 713-721. doi: 10.1038/cdd.2011. 143

Fazi, F., and Fatica, A. (2019). Interplay between N (6)-methyladenosine (m(6)A) and non-coding RNAs in cell development and cancer. Front. Cell Dev. Biol. 7:116. doi: $10.3389 /$ fcell.2019.00116

Feng, C., Liu, H., Yang, M., Zhang, Y., Huang, B., and Zhou, Y. (2016). Disc cell senescence in intervertebral disc degeneration: causes and molecular pathways. Cell Cycle 15, 1674-1684. doi: 10.1080/15384101.2016.1152433

\section{DATA AVAILABILITY STATEMENT}

The original contributions presented in the study are included in the article/supplementary material, further inquiries can be directed to the corresponding author/s.

\section{ETHICS STATEMENT}

The studies involving human participants were reviewed and approved by The Affiliated Shanghai General Hospital of Nanjing Medical University. The patients/participants provided their written informed consent to participate in this study.

\section{AUTHOR CONTRIBUTIONS}

$\mathrm{HZ}$ and $\mathrm{BS}$ conceived and designed the work. BS, LZ, and GZ performed the research and collected and analyzed the data. HZ and QS collected human tissue samples and wrote the manuscript. BS, LZ, and QS provided technical assistance. All authors read and approved the final manuscript.

Feng, R., Chen, X., Yu, Y., Su, L., Yu, B., Li, J., et al. (2010). miR-126 functions as a tumour suppressor in human gastric cancer. Cancer Lett. 298, 50-63. doi: 10.1016/j.canlet.2010.06.004

Freburger, J. K., Holmes, G. M., Agans, R. P., Jackman, A. M., Darter, J. D., Wallace, A. S., et al. (2009). The rising prevalence of chronic low back pain. Arch. Intern. Med. 169, 251-258. doi: 10.1001/archinternmed.2008.543

Frost, B. A., Camarero-Espinosa, S., and Foster, E. J. (2019). Materials for the spine: anatomy, problems, and solutions. Materials (Basel) 12:253. doi: 10.3390/ ma12020253

Froud, R., Patterson, S., Eldridge, S., Seale, C., Pincus, T., Rajendran, D., et al. (2014). A systematic review and meta-synthesis of the impact of low back pain on people's lives. BMC Musculosk. Disord. 15:50. doi: 10.1186/1471-2474-15-50

Gupta, S., Silveira, D. A., and Mombach, J. C. M. (2020). ATM/miR-34a-5p axis regulates a p21-dependent senescence-apoptosis switch in non-small cell lung cancer: a Boolean model of G1/S checkpoint regulation. FEBS Lett. 594, 227-239. doi: 10.1002/1873-3468.13615

Han, J., Wang, J. Z., Yang, X., Yu, H., Zhou, R., Lu, H. C., et al. (2019). METTL3 promote tumor proliferation of bladder cancer by accelerating pri-miR221/222 maturation in m6A-dependent manner. Mol. Cancer 18:110. doi: 10.1186/ s12943-019-1036-9

Han, M., Liu, Z., Xu, Y., Liu, X., Wang, D., Li, F., et al. (2020). Abnormality of m6A mRNA Methylation Is Involved in Alzheimer's Disease. Front. Neurosci. 14:98. doi: $10.3389 /$ fnins. 2020.00098

Hwang, P. Y., Chen, J., Jing, L., Hoffman, B. D., and Setton, L. A. (2014). The role of extracellular matrix elasticity and composition in regulating the nucleus pulposus cell phenotype in the intervertebral disc: a narrative review. J. Biomech. Eng. 136:021010. doi: 10.1115/1.4026360

Jian, D., Wang, Y., Jian, L., Tang, H., Rao, L., Chen, K., et al. (2020). METTL14 aggravates endothelial inflammation and atherosclerosis by increasing FOXO1 N6-methyladeosine modifications. Theranostics 10, 8939-8956. doi: 10.7150/ thno. 45178

Jiang, L., Zhang, X., Zheng, X., Ru, A., Ni, X., Wu, Y., et al. (2013). Apoptosis, senescence, and autophagy in rat nucleus pulposus cells: implications for diabetic intervertebral disc degeneration. J. Orthop. Res. 31, 692-702. doi: 10.1002/jor.22289

Jiao, S., Li, J., Liu, B., Yang, M., Xiu, J., and Qu, D. (2018). Nucleus pulposus cell apoptosis is attenuated by CDMP-2 through regulating oxidative damage under the hyperosmotic environment. Biosci. Rep. 38:BSR20181176. doi: 10. 1042/BSR20181176 
Kang, L., Yang, C., Yin, H., Zhao, K., Liu, W., Hua, W., et al. (2017). MicroRNA-15b silencing inhibits IL-1 $\beta$-induced extracellular matrix degradation by targeting SMAD3 in human nucleus pulposus cells. Biotechnol. Lett. 39, 623-632. doi: 10.1007/s10529-016-2280-3

Kepler, C. K., Ponnappan, R. K., Tannoury, C. A., Risbud, M. V., and Anderson, D. G. (2013). The molecular basis of intervertebral disc degeneration. Spine J. 13, 318-330. doi: 10.1016/j.spinee.2012.12.003

Lee, S. H., Lee, J. H., Lee, H. Y., and Min, K. J. (2019). Sirtuin signaling in cellular senescence and aging. BMB Rep. 52, 24-34. doi: 10.5483/BMBRep.2019.52.1. 290

Li, F., Zhang, C., and Zhang, G. (2019). m6A RNA methylation controls proliferation of human glioma cells by influencing cell apoptosis. Cytogenet. Genome Res. 159, 119-125. doi: 10.1159/000499062

Li, G., Luna, C., Qiu, J., Epstein, D. L., and Gonzalez, P. (2009). Alterations in microRNA expression in stress-induced cellular senescence. Mech. Ageing Dev. 130, 731-741. doi: 10.1016/j.mad.2009.09.002

Li, P., Gan, Y., Xu, Y., Song, L., Wang, L., Ouyang, B., et al. (2017). The inflammatory cytokine TNF-alpha promotes the premature senescence of rat nucleus pulposus cells via the PI3K/Akt signaling pathway. Sci. Rep. 7:42938. doi: $10.1038 /$ srep42938

Li, Q., Li, X., Tang, H., Jiang, B., Dou, Y., Gorospe, M., et al. (2017). NSUN2-mediated m5C methylation and METTL3/METTL14-mediated m6A methylation cooperatively enhance p21 translation. J. Cell Biochem. 118, 25872598. doi: $10.1002 / j \mathrm{cb} .25957$

Li, Z., Chen, X., Xu, D., Li, S., Chan, M. T. V., and Wu, W. K. K. (2019). Circular RNAs in nucleus pulposus cell function and intervertebral disc degeneration. Cell Prolif. 52:e12704. doi: 10.1111/cpr.12704

Li, Z., Li, X., Chen, C., Li, S., Shen, J., Tse, G., et al. (2018). Long non-coding RNAs in nucleus pulposus cell function and intervertebral disc degeneration. Cell Prolif. 51:e12483. doi: 10.1111/cpr.12483

Li, Z., Yu, X., Shen, J., Chan, M. T., and Wu, W. K. (2015). MicroRNA in intervertebral disc degeneration. Cell Prolif. 48, 278-283. doi: 10.1111/cpr. 12180

Luoma, K., Riihimaki, H., Luukkonen, R., Raininko, R., Viikari-Juntura, E., and Lamminen, A. (2000). Low back pain in relation to lumbar disc degeneration. Spine (Phila Pa 1976) 25, 487-492. doi: 10.1097/00007632-20000215000016

Ma, J. Z., Yang, F., Zhou, C. C., Liu, F., Yuan, J. H., Wang, F., et al. (2017). METTL14 suppresses the metastatic potential of hepatocellular carcinoma by modulating N(6) -methyladenosine-dependent primary MicroRNA processing. Hepatology 65, 529-543. doi: 10.1002/hep. 28885

Maes, O. C., Sarojini, H., and Wang, E. (2009). Stepwise up-regulation of microRNA expression levels from replicating to reversible and irreversible growth arrest states in WI-38 human fibroblasts. J. Cell Physiol. 221, 109-119. doi: $10.1002 /$ jcp. 21834

Morgan, P., Spiridonov, S., Goebel, R., Nissi, M., Frei, R., and Ellermann, J. (2014). MR Imaging with $\mathrm{T} 2 *$ - mapping for improved acetabular cartilage assessment in FAI-a case report with arthroscopic correlation. Orthop. Traumatol. Surg. Res. 100, 971-973. doi: 10.1016/j.otsr.2014.09.014

Nakazawa, K. R., Walter, B. A., Laudier, D. M., Krishnamoorthy, D., Mosley, G. E., Spiller, K. L., et al. (2018). Accumulation and localization of macrophage phenotypes with human intervertebral disc degeneration. Spine J. 18, 343-356. doi: 10.1016/j.spinee.2017.09.018

Pattappa, G., Li, Z., Peroglio, M., Wismer, N., Alini, M., and Grad, S. (2012). Diversity of intervertebral disc cells: phenotype and function. J. Anat. 221, 480-496. doi: 10.1111/j.1469-7580.2012.01521.x

Purmessur, D., Walter, B. A., Roughley, P. J., Laudier, D. M., Hecht, A. C., and Iatridis, J. (2013). A role for TNFalpha in intervertebral disc degeneration: a non-recoverable catabolic shift. Biochem. Biophys. Res. Commun. 433, 151-156. doi: 10.1016/j.bbrc.2013.02.034

Stojanovic, S. D., Fiedler, J., Bauersachs, J., Thum, T., and Sedding, D. G. (2020). Senescence-induced inflammation: an important player and key therapeutic target in atherosclerosis. Eur. Heart J. 41, 2983-2996. doi: 10.1093/eurheartj/ ehz919
Wang, C., Yu, X., Yan, Y., Yang, W., Zhang, S., Xiang, Y., et al. (2017). Tumor necrosis factor-alpha: a key contributor to intervertebral disc degeneration. Acta Biochim. Biophys. Sin. (Shanghai) 49, 1-13. doi: 10.1093/abbs/gmw112

Wang, X., Chen, N., Du, Z., Ling, Z., Zhang, P., Yang, J., et al. (2020). Bioinformatics analysis integrating metabolomics of $\mathrm{m}(6) \mathrm{A}$ RNA microarray in intervertebral disc degeneration. Epigenomics 12, 1419-1441. doi: 10.2217/epi-2020-0101

Wang, Y., Wang, H., Zhuo, Y., Hu, Y., Zhang, Z., Ye, J., et al. (2020). SIRT1 alleviates high-magnitude compression-induced senescence in nucleus pulposus cells via PINK1-dependent mitophagy. Aging (Albany N. Y.) 12, 16126-16141. doi: 10.18632/aging.103587

Weng, H., Huang, H., Wu, H., Qin, X., Zhao, B. S., Dong, L., et al. (2018). METTL14 inhibits hematopoietic stem/progenitor differentiation and promotes leukemogenesis via mRNA m(6)A modification. Cell Stem Cell 22, 191-205.e199. doi: 10.1016/j.stem.2017.11.016

Wu, Z., Shi, Y., Lu, M., Song, M., Yu, Z., Wang, J., et al. (2020). METTL3 counteracts premature aging via m6A-dependent stabilization of MIS12 mRNA. Nucleic Acids Res. 48, 11083-11096. doi: 10.1093/nar/gkaa816

Xia, W., Chen, H., Chen, D., Ye, Y., Xie, C., and Hou, M. (2020). PD-1 inhibitor inducing exosomal miR-34a-5p expression mediates the cross talk between cardiomyocyte and macrophage in immune checkpoint inhibitorrelated cardiac dysfunction. J. Immunother. Cancer 8:e001293. doi: 10.1136/jitc2020-001293

Xiang, Q., Kang, L., Wang, J., Liao, Z., Song, Y., Zhao, K., et al. (2020). CircRNACIDN mitigated compression loading-induced damage in human nucleus pulposus cells via miR-34a-5p/SIRT1 axis. EBioMedicine 53:102679. doi: 10 . 1016/j.ebiom.2020.102679

Xie, L., Huang, W., Fang, Z., Ding, F., Zou, F., Ma, X., et al. (2019). CircERCC2 ameliorated intervertebral disc degeneration by regulating mitophagy and apoptosis through miR-182-5p/SIRT1 axis. Cell Death Dis. 10:751. doi: 10.1038/ s41419-019-1978-2

Yang, J., Liu, J., Zhao, S., and Tian, F. (2020). N(6)-methyladenosine METTL3 Modulates the proliferation and apoptosis of lens epithelial cells in diabetic cataract. Mol. Ther. Nucleic Acids 20, 111-116. doi: 10.1016/j.omtn.2020.02.002

Zhang, B. Y., Han, L., Tang, Y. F., Zhang, G. X., Fan, X. L., Zhang, J. J., et al. (2020). METTL14 regulates M6A methylation-modified primary miR-19a to promote cardiovascular endothelial cell proliferation and invasion. Eur. Rev. Med. Pharmacol. Sci. 24, 7015-7023. doi: < doi>

Zhang, C., Fu, J., and Zhou, Y. (2019). A review in research progress concerning m6A methylation and immunoregulation. Front. Immunol. 10:922. doi: 10. 3389/fimmu.2019.00922

Zhang, Z., Kakutani, K., Maeno, K., Takada, T., Yurube, T., Doita, M., et al. (2011). Expression of silent mating type information regulator 2 homolog 1 and its role in human intervertebral disc cell homeostasis. Arthritis Res. Ther. 13:R200. doi: 10.1186/ar3533

Zhao, C. Q., Jiang, L. S., and Dai, L. Y. (2006). Programmed cell death in intervertebral disc degeneration. Apoptosis 11, 2079-2088. doi: 10.1007/ s10495-006-0290-7

Zhou, X., Chen, L., Grad, S., Alini, M., Pan, H., Yang, D., et al. (2017). The roles and perspectives of microRNAs as biomarkers for intervertebral disc degeneration. J. Tissue Eng. Regen. Med. 11, 3481-3487. doi: 10.1002/term.2261

Zhu, J., Zhang, X., Gao, W., Hu, H., Wang, X., and Hao, D. (2019). lncRNA/circRNAmiRNAmRNA ceRNA network in lumbar intervertebral disc degeneration. Mol. Med. Rep. 20, 3160-3174. doi: 10.3892/mmr.2019.10569

Conflict of Interest: The authors declare that the research was conducted in the absence of any commercial or financial relationships that could be construed as a potential conflict of interest.

Copyright (c) 2021 Zhu, Sun, Zhu, Zou and Shen. This is an open-access article distributed under the terms of the Creative Commons Attribution License (CC BY). The use, distribution or reproduction in other forums is permitted, provided the original author(s) and the copyright owner(s) are credited and that the original publication in this journal is cited, in accordance with accepted academic practice. No use, distribution or reproduction is permitted which does not comply with these terms. 